\title{
¿Se puede prevenir el parto prematuro por infección bacteriana ascendente y sus resultados adversos en los hospitales públicos de Chile?
}

\author{
Alfredo Ovalle ${ }^{1}$, María Angélica Martínez ${ }^{\dagger 2}$ y Jorge Figueroa' ${ }^{1}$
}

\footnotetext{
Servicio y Departamento de

Obstetricia, Ginecología y Neonatología, Hospital Clínico San Borja Arriarán, Santiago, Chile. Facultad de Medicina, Universidad de Chile, Santiago,

2Programa de Microbiologia Micología, Facultad de Medicina Universidad de Chile, Santiago,

Los autores no tienen conflicto de interés.

El manuscrito no requirió financiamiento.

Recibido: 26 de junio de 2018 Aceptado: 5 de marzo de 2019

Correspondencia a: Alfredo Ovalle Salas alfredoovalle@gmail.com
}

\section{Can preterm birth be prevented from ascending bacterial infection and its adverse results in the public hospitals of Chile?}

Preterm birth (PB) is the main contributor to the perinatal morbidity/mortality. In spite of the knowledge of the risk factors and the introduction of medical interventions intended to prevent $\mathrm{PB}$, its frequency has increased. Ascending bacterial infection (ABI) is the obstetric condition most frequently associated to PB causing an important adverse perinatal outcome in a public hospital in Chile. This review shows the association between PB and $\mathrm{ABI}$, analyzes the physiopathology and immunology of vaginal infections in the susceptible pregnant woman., as well as their application in this group of effective measures demonstrated by evidence, such as routine control, treatment of genitourinary tract infections (GTI), prophylactic or therapeutic cerclage, use of probiotics, use of vaginal progesterone, metabolic control of diabetes mellitus and weight of the obese woman. Treatment GTI together with the use of medical interventions that improve the vaginal immunity in the risk population allow to predict a reduction of $\mathrm{PB}$ by $\mathrm{ABI}$ and of its immediate consequences, long term sequels and high associated costs, with the consequent benefit of the public health in Chile.

Keywords: Preterm birth; ascending infection; chorioamnionitis; preterm birth prevention

Palabras clave: Parto prematuro; infección ascendente; corioamnionitis; prevención nacimiento prematuro.

\section{Introducción}

$\mathrm{E}$ 1 parto prematuro (PP) es uno de los problemas de Salud Pública más importantes de la Obstetricia en todo el mundo ${ }^{1}$. Es el principal contribuyente de la morbilidad neonatal, de las complicaciones neurológicas y respiratorias a largo plazo, de la mortalidad perinatal y de los costos elevados por la prolongada atención que suelen tener los niños prematuros en las Unidades de Cuidados Intensivos neonatales ${ }^{1,2}$.

En las Guías actuales de prevención del PP, los factores de riesgo asociados comúnmente mencionados son: raza negra de la madre, PP previo, infección genitourinaria (IGU), tabaquismo, bajo peso/obesidad, depresión materna, estrés, mala alimentación, fertilidad asistida, enfermedad periodontal y embarazo múltiple ${ }^{2-4}$. En su prevención, cinco intervenciones predijeron una reducción de nacimientos prematuros: dejar de fumar, disminución de transferencias múltiples de embriones durante las tecnologías de reproducción asistida, cerclaje cervical, suplementos de progesterona, reducción de la inducción del parto sin indicación médica y del parto por cesárea ${ }^{4}$. A pesar de los avances en el conocimiento de los factores de riesgo y de la introducción de intervenciones médicas destinadas a la prevención del nacimiento prematuro, su frecuencia ha aumentado en todo el mundo ${ }^{1-6}$.

El camino a seguir según recomendación de un artículo publicado en la revista The Lancet incluye elaborar una clasificación apropiada de las causas del parto prematuro, que permita mejorar el diagnóstico, estratificar los riesgos y desarrollar intervenciones innovadoras de prevención basadas en una mejor comprensión de los estudios etiológicos y genéticos subyacentes ${ }^{4}$.

En este mismo sentido en el Hospital Clínico San Borja Arriarán (HCSBA) ideamos un método que identifica los factores de riesgo o condiciones obstétricas relevantes de PP, basado en el análisis de los datos clínicos maternos, resultados de laboratorio y hallazgos patológicos placentarios. El principal factor de riesgo encontrado con esta clasificación es la infección bacteriana ascendente (IBA). En este hospital, la IBA es responsable de la mayoría de los PPs y de la morbilidad/mortalidad perinatal asociada $^{7}$. Identificar este grupo vulnerable, pesquisar, tratar infecciones del tracto urinario (ITU) y de vagina y realizar otras intervenciones específicas en estas mujeres embarazadas probablemente permitan disminuir el PP por $\mathrm{IBA}^{3,4,7-11}$ 


\section{Parto prematuro en el mundo}

Las cifras de nacimiento prematuro (antes de las 37 semanas cumplidas) en países con muy alto índice de desarrollo humano (según definición de las Naciones Unidas) en 2010, oscilan entre 5,3\% (Letonia), 5,5\% (Croacia), 7,1\% (Chile), hasta 14,7\% (Chipre), 14,0\% (Baréin), $12,0 \%$ (E.U.A.) y cada una de estas cifras son más altas que los años previos ${ }^{4,5}$. Las tasas se han incrementado por el crecimiento de los PPs por indicación médica, por inducción del parto sin indicación médica, por aumento de las cesáreas y de los embarazos múltiples concebidos artificialmente ${ }^{2-7}$.

\section{Parto prematuro en el HCSBA}

La frecuencia es ascendente 7\% (2007), 9\% (2010), $11 \%$ (2011), 13,8\% (2014) y 11\% (2017) (Sistema Informático Perinatal. OPS/OMS CLAP, datos no publicados). Este ascenso es principalmente por incremento del nacimiento prematuro espontáneo asociado con IBA ${ }^{7}$. Este factor de riesgo, el más frecuente en el HCSBA, mencionado ocasionalmente en los países desarrollados, está vinculado con la población de menores recursos. En nuestro hospital, la mayor morbilidad/mortalidad del PP se concentra en el grupo nacido bajo las 34 semanas (PP temprano $)^{12}$. La muerte fetal en el nacimiento prematuro temprano y tardío (34 a 36,6 semanas) representan 66,3 y $12 \%$, respectivamente de todos los mortinatos del servicio $^{13}$. La mortalidad perinatal es $82,7 \%$ entre $22-25,6$ semanas, $42,3 \%$ entre $26-29.6$ semanas y $13,9 \%$ entre 30 y 34 semanas $^{12}$.

\section{Factores asociados con el PP en el HCSBA}

En los últimos 10 años usamos el método que identifica los factores de riesgo o condiciones obstétricas relevantes de $\mathrm{PP}^{7}$ basado en el análisis de los datos clínicos maternos, resultados de laboratorio y hallazgos patológicos placentarios. La IBA es el factor de riesgo más frecuentemente asociado al PP temprano en gestaciones únicas, identificada con este método.

Identificación del PP (embarazos únicos) por IBA como factor riesgo, mediante método usado en el HCSBA.

El método utilizado considera como PP originado por IBA, al embarazo con a) datos clínicos (uno o varios): elevada frecuencia de invasión microbiana de la cavidad amniótica (IMCA) encontrada en las siguientes condiciones obstétricas: rotura prematura de membranas (RPM) en el embarazo de pretérmino $(54 \%)^{14,15}$, sangrado vaginal "idiopático" $(14 \%)^{16}$ con o sin desprendimiento amniocorial ${ }^{7,16}$, membranas prolapsadas bajo el orificio cervical externo en el embarazo de pretérmino $(51,5 \%)^{17}$, desprendimiento prematuro de placenta normoinserta
(DPPNI) $(7 \%)^{7,15}$, corioamnionitis clínica, pacientes sin amenaza de PP y cérvix menor de $15 \mathrm{~mm}$ medido por ultrasonografía $(4 \%)^{18}$, b) datos de laboratorio: presencia de IGU: vaginosis bacteriana (VB) o vaginitis aeróbica (VA) por Streptococcus agalactiae (Streptococcus grupo B) (SGB) y/o ITU ${ }^{7}$ y c) hallazgos placentarios, lesiones agudas propias de infección: corioamnionitis histológica (CH), funisitis, intervellositis ${ }^{7,14,15}$.

$\mathrm{CH}$ aguda y funisitis aguda son hallazgos patognomónicos de infección. Confirman esta relación las siguientes evidencias. En una serie de PP con RPM, las pacientes con IMCA por SGB o con IMCA por otras bacterias únicas o asociadas, tuvieron más frecuentemente $\mathrm{CH}$ $(100 \%, 88 \%)$ y funisitis $(64 \%, 80 \%)$ respectivamente, que las pacientes sin IMCA y $\sin$ ICV $(17 \%, 4 \%)$, respectivamente $p<0,0001^{14}$. En otra serie de PP con RPM, la $\mathrm{CH}$ se encontró más frecuentemente en las pacientes con infección cérvico-vaginal (ICV) $(73,6 \%)$, que en las sin ICV $(11,1 \%) \mathrm{p}<0,0001$, lo que sugiere que la $\mathrm{CH}$ es una infección intraamniótica, producto de infección ascendente desde la vagina ${ }^{15}$.

\section{Parto prematuro temprano (22-34 semanas) por IBA en embarazos únicos en el HCSBA}

Es responsable de un tercio de los PP entre 22-34 semanas (36\%), de $51 \%$ de los PP tempranos espontáneos, de 52 y $73,1 \%$ de los nacimientos menores de 30 y 26 semanas, respectivamente ${ }^{7}$. La infección vaginal o urinaria por SGB y el sangrado vaginal, con o sin desprendimiento amniocorial (19\%), son las condiciones clínicas más comunes presentes en estos casos?

La IBA es el factor de riesgo más frecuentemente asociado con la mortalidad perinatal del PP temprano (41\%). Las muertes ocurrieron, en $97 \%$ de los casos, antes de las 30 semanas y fueron, fetales en $30 \%$ y neonatales precoces en $70 \%{ }^{12}$. Este factor de riesgo se identificó mediante el método descrito anteriormente, más autopsia fetal o neonatal. Las causas del fallecimiento perinatal fueron neumonía congénita, preferentemente por SGB (fetal o connatal dentro de las primeras $24 \mathrm{~h}$ de vida), hipoxia aguda secundaria a un DPPNI y complicaciones propias de la prematuridad extrema (síndrome de dificultad respiratoria, hemorragia intraventricular, enterocolitis necrosante). En todas las placentas de estos niños con muerte perinatal se encontró $\mathrm{CH}$ y en la mayoría funisitis.

\section{Infección genital ascendente causante de parto prematuro}

La microbiota vaginal normal de mujeres sanas asintomáticas en edad reproductiva está formada mayori- 
tariamente por comunidades microbianas con predominio de especies de Lactobacillus, siendo L. crispatus, L. gasseri L. iners y L. jensenii las más frecuentes ${ }^{19,20}$. Los lactobacilos protegen la función de barrera de la mucosa vaginal mediante fenómenos de exclusión microbiana que incluyen la adherencia, la producción de compuestos antimicrobianos $\mathrm{y}$, especialmente, la de ácido láctico ${ }^{21}$. La pérdida o disminución de lactobacilos es un factor determinante en el cambio de microbiota normal a microbiota vaginal alterada ${ }^{20,22}$, que conduce al desarrollo de infecciones por disbiosis, como vaginosis bacteriana (VB) y vaginitis aeróbica (VA), favoreciendo el PP y otras complicaciones reproductivas ${ }^{23-27}$. Otra característica es la ausencia de inflamación, medida por el recuento de leucocitos polimorfonucleares $<$ de 10 por campo $400 \mathrm{x}^{25-27}$.

Vaginosis bacteriana es la más frecuente de las IBA. Afecta a entre 5 y $26 \%$ de las mujeres embarazadas ${ }^{28-30}$. Microbiológicamente se caracteriza por la pérdida de especies de lactobacilos y su reemplazo por una microbiota de mayor diversidad y complejidad. Las técnicas de cultivo han identificado un bajo número de especies, las más frecuentes son, Gardnerella vaginalis, bacterias anaerobias: Bacteroides spp., Prevotella spp., cocáceas grampositivas, Mobiluncus spp. y Mycoplasma homi$n i s^{27,31,32}$. Con el empleo de técnicas de secuenciación de los ácidos nucleicos de nueva generación y herramientas de bioinformática de alta resolución, se ha confirmado que las muestras con VB contienen comunidades vaginales heterogéneas diversas y que las muestras sin VB están colonizadas principalmente por lactobacilos ${ }^{33}$. Además, se ha podido detectar la existencia de subgrupos, los que presentan asociaciones interespecies con correlaciones que sugieren probables dependencias metabólicas ${ }^{33}$. Del punto de vista clínico se ha demostrado asociaciones específicas de los microorganismos más frecuentes de VB con uno o más de los criterios clínicos de $\mathrm{Amsel}^{33}$.

La VB se caracteriza por ausencia de signos inflamatorios en la mitad de los casos, motivo de su nombre, vaginosis. Sin embargo, las complicaciones reproductivas asociadas con VB se concentran en pacientes que habitualmente presentan inflamación (leucocitos polimorfonucleares $>$ de 10 por campo 400x) y alteración inmunológica vaginal y que corresponden a aquéllas que tienen factores de riesgo de infección, (detallados más adelante) $)^{34}$. Se postula que la presencia de un polimorfismo genético (portación del alelo 2 de FNT alfa) especialmente existente en la población afroamericana, que interactúa con VB sintomática, aumenta la inflamación y los resultados adversos ${ }^{35}$.

Vaginitis aeróbica (VA) ha sido descrita en mujeres en edad reproductiva ${ }^{36}$. Se atribuye a bacterias entéricas como Escherichia coli, Enterococcus spp., o Streptococcus agalactiae, Staphylococcus aureus o Streptococcus anginosus $^{7,20,27,30,36,37}$. Su prevalencia es menor que la VB y tiene una fuerte respuesta inflamatoria (leucocitos polimorfonucleares $>$ de 10 por campo 400x). Se ha asociado con mayor frecuencia con PP,20,27,30,36,37, de preferencia menor de 30 semanas, con morbilidad/mortalidad perinatal, principalmente por SGB en nacidos menores de 30 semanas $^{7,12}$ y por $E$. coli en menor medida ${ }^{36,38}$.

La importancia de la respuesta inflamatoria aumentada de VB y de VA en el incremento del nacimiento prematuro se corrobora por las siguientes evidencias. Los niveles de RANTES (regulated on activation, normal $T$-cell expressed and secreted) y de IL- $1 \beta$ encontrados en el líquido cérvico-vaginal a mediados del $2^{\circ}$ trimestre del embarazo, se correlacionan con la fibronectina fetal cuantitativa. Estos niveles disminuyen significativamente en las mujeres cuyo parto es a término en comparación con aquéllas que tienen parto prematuro. Esta observación sugiere que el PP puede caracterizarse por inflamación corio-decidual mantenida, la que de confirmarse, puede tener valor clínico para el cribado del $\mathrm{PP}^{39}$.

En mujeres embarazadas con inflamación endocervical (leucocitos polimorfonucleares $>$ de 10 por campo 400x) con trabajo de PP, membranas intactas y sin infección de la cavidad amniótica, tratadas con clindamicinagentamicina, en sub-análisis, se encontró una menor tasa de morbilidad/mortalidad neonatal compuesta en comparación con las que recibieron placebo. Este hallazgo, que debe confirmarse, sugiere la importancia de la inflamación en el resultado adverso del PP y la necesidad de tratar la ICV oportunamente para la prevención del nacimiento anticipado y de sus consecuencias ${ }^{40}$.

Microbiota intermedia (MI) es una categoría de microbiota descrita por métodos microscópicos de diagnóstico $^{41,42}$. Su microbiología no está aún bien caracterizada y en algunos casos se observa como parte de un continuo entre microbiota normal y VB, con cantidades variables de Gardnerella y Prevotella vs Lactobacillus spp., mientras que, en otros casos, se observan morfotipos de bacterias de origen entérico, sugiriendo un origen independiente ${ }^{20}$. Del punto de vista clínico, se ha demostrado que mujeres embarazadas con MI y ausencia o muy bajo recuento de Lactobacillus spp tienen un riesgo significativamente más alto de nacimientos prematuros que mujeres con MI con presencia de Lactobacillus spp. ${ }^{20,43}$. Si esta caracterización microbiológica se presenta en pacientes con riesgo de infección que tienen inflamación vaginal aumentada se incrementa el riesgo de $\mathrm{PP}^{34,36}$.

Micoplasmas genitales. Ureaplasma spp. y $M$. hominis son los micoplasmas genitales más frecuentemente aislados del tracto genital femenino ${ }^{44}$ con tasas de colonización de 57 a $82 \%$ y 7,3 a 50\%, respectivamente $^{45}$. En las mujeres embarazadas, la colonización aumenta significativamente a lo largo del embarazo, debido probablemente al efecto de los estrógenos ${ }^{44,45}$. Los ureaplasmas comprenden dos especies: Ureaplas- 
ma urealyticum y Ureaplasma parvum, siendo ésta la especie la más frecuente, tanto en muestras vaginales de mujeres sanas asintomáticas, como del LA de pacientes con complicaciones del embarazo ${ }^{46}$.

Existe controversia referente a la utilidad de la pesquisa y tratamiento de Ureaplasma spp en muestras vaginales para reducir el PP. Evidencias a favor de asociación con PP: a) constituyen los microorganismos más frecuentes aislados en vagina y en líquido amniótico (LA) en el PP ${ }^{25,46}$; b) invaden el saco amniótico e inducen respuesta inflamatoria con corioamnionitis y $\mathrm{PP}^{44}$; c) existe asociación entre colonización del tracto respiratorio neonatal y displasia broncopulmonar en recién nacidos $(\mathrm{RN})<$ de $1.500 \mathrm{~g}^{47}$. Evidencias en contra de esta asociación son: a) el tratamiento con eritromicina de la colonización genital por ureaplasmas en el embarazo no reduce los $\mathrm{RN}<$ de $2.500 \mathrm{~g}^{48}$; b) el aislamiento de Ureaplasma spp en LA mediante técnica molecular, reacción de polimerasa en cadena (RPC) en mujeres embarazadas sanas y gestación $<$ de 20 semanas con fines genéticos, no se asoció con RPM, con PP ni con resultado neonatal adverso ${ }^{49}$; c) no se encontró relación entre Ureaplasma spp aislado en microbiota vaginal anormal durante el primer trimestre del embarazo y el nacimiento prematuro ${ }^{26}$.

Creemos que existe asociación entre Ureaplasma spp y $\mathrm{PP}^{25,46}$, pero no hay necesidad de pesquisa rutinaria por las siguientes evidencias: a) En serie de PP con RPM, cuando existió IMCA por Ureaplasma spp (habitualmente asociado a otros microorganismos), en la ICV también se encontró en $92 \%$ asociación de esta bacteria con microbiota anormal, VB y en menor medida con VA y MI ${ }^{14}$. Sugiere que el ascenso de Ureaplasma spp al interior de la cavidad amniótica requiere asociación con estas infecciones bacterianas; b) En otra serie de PP con RPM, el uso de clindamicina-gentamicina intravenosa aumentó significativamente el intervalo desde la randomización al parto (12,1 días de promedio) en comparación con grupo placebo (7,8 días) $\mathrm{p}<0,05$. Este efecto fue más fuerte en pacientes con ICV y sin IMCA, que en pacientes con $\mathrm{ICV}$ e IMCA ${ }^{50}$. Este resultado sugiere que el beneficio de clindamicina-genatmicina se debería a la erradicación total o parcial de la microbiota anormal incluyendo Ureaplasma spp; c) Clindamicina oral usada en el $2^{\circ}$ trimestre en pacientes con microbiota vaginal anormal y $\mathrm{VB}$, redujo el PP $<$ de 37 semanas y el aborto tardío ${ }^{51}$. Posiblemente, la reducción de la inflamación y la eliminación de la microbiota vaginal anormal de Ureaplasma spp y de otras bacterias influyen en este logro.

En base a estos resultados, no recomendamos pesquisa rutinaria de Ureaplasma spp. Sería suficiente la pesquisa de VB, de VA, de MI y tratamiento de estas infecciones.

Mycoplasma hominis se asocia con VB (60\%), tiene acción sinergística con $G$. vaginalis y depende de nutrien- tes producidos por anaerobios. Por su asociación con VB bastaría con el diagnóstico de esta entidad microbiológica. Aislada en vagina en pacientes de riesgo se trata como todas las VB sintomáticas. Se relaciona con PP cuando se asocia con microbiota vaginal anormal y especialmente $\mathrm{VB}^{25,27,46}$.

\section{Infecciones de transmisión sexual}

Neisseria gonorrhoeae y Trichomonas vaginalis se asocian con PP pero es incierta la relación de Chlamydia trachomatis con nacimiento prematuro ${ }^{25,27,46,50-52}$. La pesquisa de estas ITS se recomienda en mujeres embarazadas con conducta de riesgo y no rutinariamente, aunque $T$. vaginalis se detecta habitualmente por el examen directo al fresco.

\section{Factores de riesgo de IBA}

El método utilizado en el HCSBA que identifica los factores de riesgo de IBA se basa en la siguiente evidencia. En mujeres embarazadas, con y sin IGU (ITU y/o ICV) recurrentes, se estudiaron los antecedentes mórbidos, infecciones, resultados adversos obstétricos o enfermedades que sugieren alteración del sistema inmune. Se encontraron: aborto espontáneo de segundo trimestre asociado con IGU, ICV a repetición (tres o más episodios), ITU recurrente, PP asociado con IGU, diabetes mellitus familiar y depresión ${ }^{9,27,53}$.

La mayor frecuencia de IGU y de sus consecuencias (aborto y parto prematuro), de la población de riesgo, pueden ser explicadas por las siguientes evidencias que demuestran alteración de la inmunidad.

\section{Alteración del sistema inmune innato (SII) cérvico- vaginal favorecedor de IGU y de sus consecuencias}

En el tracto genital femenino se encuentran los componentes de la respuesta inmune innata (RII), como los receptores de reconocimiento de moléculas microbianas tipo Toll (TLRs) y los péptidos antimicrobianos $(\mathrm{AMPs})^{54,55}$. La producción adecuada de todos estos componentes cumple un rol protector apropiado a las necesidades de cada segmento. La estimulación de los TLRs lleva a la activación y producción de citoquinas pro-inflamatorias, quimioquinas y otros mediadores inmunes, favoreciendo un ambiente pro-inflamatorio. Los AMPs poseen actividad antimicrobiana de amplio espectro, pero además son moduladores de la respuesta inflamatoria, promoviendo un ambiente anti-inflamatorio. En VB se induce la producción de IL-1, sin cambios en las concentraciones de IL- $8^{56-58}$. Hay disminución de las concentraciones de varios AMPs, como la defensina HBD- $3^{59}$, las antiproteasas SLPI y la elafina ${ }^{54,57}$. En una serie de mujeres embarazadas con VB, principalmente afroamericanas, muestras tomadas en primer y segundo 
trimestre tuvieron concentraciones vaginales más bajas de HBD-3, que las sin $\mathrm{VB}^{59}$. Esta pobre respuesta inmune puede explicar la menor presencia de neutrófilos en VB y sus consecuencias. Además, existen diferencias en la RII del epitelio cérvico-vaginal según la existencia de algunas especies particulares de bacterias o productos de bacterias asociadas a $\mathrm{VB}^{56}$, a factores raciales ${ }^{60}$ y entre mujeres embarazadas ${ }^{59}$, requiriéndose más estudios para comprender mejor la RII en VB.

Entre las $\beta$-defensinas, la HBD-2 se encuentra naturalmente en el LA y explica, en parte, su capacidad antibacteriana. En pacientes con PP e IMCA se incrementa en el $\mathrm{LA}^{61}$, y aumenta con la gravedad de la corioamnionitis histológica $^{62}$, mientras que los niveles de HBD-3 y elafina aumentan en mujeres con corioamnionitis clínica ${ }^{63}$.

Estos AMPs se incrementan en placenta y en membranas fetales por efecto de las citoquinas pro-inflamatorias ${ }^{63}$. La evidencia indica que los estímulos inflamatorios y la infección afectan la expresión de los AMPs en ambos segmentos del tracto genital en mujeres embarazadas. En otro ámbito, la detección de AMPs está investigándose como predictor de infección/inflamación con muy buenos resultados.

\section{Otros factores de riesgo que favorecen el aumento de infecciones por deterioro de la inmunidad}

\section{Diabetes mellitus (DM), diabetes gestacional (DG)}

Las mujeres diabéticas embarazadas tienen un mayor riesgo de ICV y de bacteriuria, incluida por SGB, mayor resistencia antimicrobiana y mayores niveles de hemoglobina glicosilada en comparación con las mujeres gestantes sin diabetes mellitus ${ }^{64}$.

\section{Depresión}

La mujer embarazada con depresión tiene mayor riesgo de infección. Este mayor riesgo se puede explicar porque se incrementa la respuesta inflamatoria durante la gestación basada en la siguiente evidencia. Las mujeres embarazadas depresivas con mayor intensidad de síntomas, vacunadas contra virus influenza, tuvieron mayor respuesta inflamatoria que las pacientes con menor intensidad de síntomas. Se sugiere que las mujeres que sufren depresión intensa tendrían una respuesta inmunológica alterada y serían más susceptibles a las infecciones durante la gestación ${ }^{65}$.

\section{Obesidad}

La mujer obesa embarazada, tiene entre 2,5 y 4,5 veces mayor riesgo que la de peso normal de presentar ICV especialmente por SGB, aborto del segundo trimestre por IBA, parto prematuro por IBA, infección del parto, puerperio y morbilidad/mortalidad perinatal por IBA durante el embarazo. La obesidad es un factor de riesgo independiente de ICV, aborto del segundo trimestre por IBA y PP por IBA ${ }^{66}$. Este mayor riesgo de infección se puede explicar porque la mujer gestante obesa posee una proporción significativamente menor de linfocitos CD8 y de células NK, una mayor proporción de linfocitos $\mathrm{B}$, producción alterada de citoquinas y deterioro de la capacidad de proliferación de los linfocitos ${ }^{67}$. En pacientes obesas la relación $\mathrm{Th} 1 / \mathrm{Th} 2$ se inclina hacia el predominio de una respuesta Th1, que promueve infiltración de macrófagos M1, en contraste con mujeres delgadas en las cuáles hay predominio de macrófagos M2. Los macrófagos se acumulan con la adiposidad, se activan permanentemente originando inflamación y resistencia a la insulina ${ }^{68}$.

\section{Lupus eritematoso sistémico (LES)}

Las mujeres embarazadas con LES tienen respectivamente 1,7 y 1,3 veces más probabilidades de tener infección (sin aumento de corioamnionitis) y de recibir antimicrobianos durante el trabajo de parto, que las gestantes sin LES. Los niños de mujeres con LES tuvieron 1,4 mayor riesgo de padecer infección durante la hospitalización neonatal, gran parte de la cual es atribuible al nacimiento prematuro ${ }^{69}$.

\section{Prevención del parto prematuro por IBA en un embarazo único}

\section{Recomendaciones}

En base al análisis fisiopatológico expuesto en los párrafos anteriores, recomendamos las siguientes intervenciones avaladas por la evidencia para disminuir el parto prematuro asociado con IBA.

\section{Detección de la población embarazada con riesgo de infección génito-urinaria en nivel primario de atención de salud, mediante factores de riesgo de IBA}

Los siguientes son factores de riesgo de infección ascendente: a) antecedente de aborto espontáneo de segundo trimestre asociado a IGU y/o con hallazgos en la biopsia placentaria de corioamnionitis, funisitis; b) antecedente de PP asociado a IGU y/o con hallazgos en la biopsia placentaria de corioamnionitis, funisitis; en el embarazo actual presencia de: c) ICV recurrente (tres episodios o más); d) ITU recurrente; e) diabetes pre y gestacional, f) depresión, g) obesidad y h) LES ${ }^{9,27,30,53,64-}$ ${ }^{66,69}$. Recomendación B (CTFPHC,SIGN) ${ }^{70}$. Las mujeres embarazadas identificadas en el primer trimestre en nivel primario con estas características deben derivarse al nivel secundario donde serán atendidas durante todo el embarazo. 


\section{Pesquisa y tratamiento de ICV e ITU en población} de riesgo mediante toma de flujo vaginal $+S G B y$ urocultivo.

Pesquisa recomendada en la mujer embarazada de riesgo: toma de flujo vaginal al término del primer trimestre, entre 22 y 24 semanas y entre 28 y 30 semanas que incluya: a) examen microscópico directo al fresco (en solución salina fisiológica $\mathrm{NaCl} 9 \%$ ); b) tinción de Gram; c) cultivo en agar sangre (muestra transportada en medio de Stuart o Amies); d) muestra vagino-rectal para aislar SGB con siembra inmediata en medio de cultivo enriquecido selectivo o mediante RPC. Se debe identificar: la microbiota normal, que incluye presencia de lactobacilos y ausencia de inflamación (leucocitos polimorfonucleares $<$ de 10 por campo 400x), la microbiota anormal o alterada, VB con inflamación, VA y $\mathrm{MI}^{26,27,30,36}$.

La pesquisa debe hacerse al término del primer trimestre, porque la ausencia de lactobacilos en la microbiota vaginal anormal en ese período se asocia más consistentemente con PP y con pérdidas del embarazo a mitad de la gestación ${ }^{26}$. Recomendación $\mathrm{A}^{70}$. Se toma muestra de flujo vaginal entre 22 y 24 y entre 28 y 30 semanas porque la mayoría de los PP y muertes perinatales por IBA, especialmente por SGB ocurren antes de las $26 \mathrm{y}$ 30 semanas, respectivamente ${ }^{7,12}$. Recomendación $\mathrm{B}^{70}$.

Tratamiento de la microbiota vaginal anormal. En revisión sistemática y meta-análisis de trabajos randomizados, se concluyó que la administración de clindamicina oral $o$ vaginal en pacientes con microbiota vaginal anormal y VB asintomática en gestaciones menores de 22 semanas disminuyó significativamente el $\mathrm{PP}<$ de 37 semanas. No redujo el $\mathrm{PP}<$ de 33 semanas, el niño de bajo y muy bajo peso al nacer, admisión a la UCI, muerte fetal e infección perinata ${ }^{51}$. Recomendación $\mathrm{A}^{70}$.

La Revisión Sistemática Cochrane (RSC), un trabajo incluido, con más de 4.000 pacientes, sugiere detección de VB, TV y candidiasis vaginal antes de las 20 semanas de gestación y tratamiento con clindamicina crema vaginal para VB, oral para VB recurrente, clotrimazol vaginal para candidiasis y metronidazol vaginal para TV. Se redujo el PP, RR 0,55 (95\% IC 0,41-0,75); el RN < de 2.500 g. RR $0,48(95 \%$ IC $0,34-0,66)$ y el $\mathrm{RN}<$ de 1.500 g. RR 0,34 $(95 \% \text { IC } 0,15-0,75)^{71}$. Recomendación $\mathrm{A}^{70}$.

Nuestras sugerencias en el tratamiento de las ICV son: clindamicina oral o metronidazol oral para tratar VB, ampicilina o clindamicina oral para tratar VA por SGB, cefalosporinas para tratar VA por E. coli y clindamicina oral para tratar MI, metronidazol oral para tratar TV, recomendaciones contenidas en Guía Perinatal ${ }^{27,30}$ o en Guías locales $^{51}$. Recomendación $\mathrm{A}^{70}$. La ausencia de lactobacilos será tratada con probióticos (ver más adelante). Es necesario hacer nuevos trabajos prospectivos randomizados para demostrar que los tratamientos sugeridos usados en población de riesgo de infección reducen el PP.
Toma de sedimento de orina segundo chorro y urocultivo durante el primer trimestre, entre 22 y 24 semanas y entre 28 y 30 semanas. La pesquisa se debe hacer en estas semanas por los mismos fundamentos dados para el cribado de las ICV.

Según la RSC, el tratamiento antimicrobiano de la bacteriuria asintomática en el embarazo redujo el riesgo de pielonefritis, el bajo peso al nacer y el nacimiento prematuro, pero esta asociación debe interpretarse con precaución por la mala calidad de los estudios incluidos ${ }^{72}$. Recomendación $\mathrm{A}^{70}$. No obstante esta cautela, creemos que el tratamiento de las ITU reduce el PP porque trata la ICV simultáneamente. Nuestra opinión se fundamenta en el estudio que dice, las mujeres embarazadas con pielonefritis por $E$. coli tienen frecuentemente (65\%) VA por $E$. $\operatorname{coli}^{73}$. La infección vaginal por similar microorganismo aislado en la ITU sugiere ascenso de esta bacteria desde la vagina no sólo hacia la vía urinaria sino también hacia el interior del útero. Por consiguiente, el tratamiento de la ITU eliminaría la bacteria, tanto de la orina como de la vagina, reduciendo el riesgo de PP.

Tratamiento de las ITU de acuerdo a las recomendaciones contenidas en la Guía Perinatal ${ }^{9}$ o en Guías locales. Es necesario hacer nuevos trabajos prospectivos randomizados para demostrar que los tratamientos usados en mujeres gestantes con riesgo de IBA, con ITU e ICV concomitante reducen el PP.

\section{Cerclaje profiláctico en pacientes con antecedente de abortos de segundo trimestre y/o partos prematuros por IBA, demostrados por biopsia placentaria y sin hijos vivos realizado en el primer trimestre \\ El cerclaje previene el nacimiento prematuro y reduce} la mortalidad perinatal y la morbilidad compuesta en mujeres gestantes con embarazo único, PP previos y longitud cervical $<25 \mathrm{~mm}^{74}$. Recomendación $\mathrm{A}^{70}$.

El cerclaje cervical según la RSC, reduce el riesgo de PP y probablemente el riesgo de muerte perinatal en mujeres con embarazo único y alto riesgo de PP. Recomendación $\mathrm{A}^{70}$. No hay diferencia del efecto del cerclaje en base a antecedentes obstétricos o indicaciones con cuello uterino acortado $^{75}$.

En nuestra serie de pacientes con embarazos únicos y antecedente de PPs por IBA, el cerclaje profiláctico redujo el PP. Realizado en el primer trimestre en mujeres embarazadas sin acortamiento cervical y con el antecedente de PP o aborto de segundo trimestre por IBA, comparado con el manejo de mujeres gestantes con similares antecedentes y sin cerclaje, que mantuvieron longitud cervical $>25 \mathrm{~mm}$ durante el segundo trimestre, el cerclaje redujo el nacimiento prematuro $<37$ semanas (OR $0.08,95 \%$ IC $0,09-0,70$ ), el nacimiento prematuro $<34$ semanas (OR $0,11,95 \%$ IC $0,01-0,99$ ) y la CH (OR $0,01,95 \%$ IC $0,01-0,83)^{76}$. El reparo de este trabajo es el 
posible sesgo existente en la distribución de las pacientes en los grupos.

La reducción de la $\mathrm{CH}$ en nuestra serie, sugiere que el cerclaje posiblemente dificulta el ascenso de la infección y de la inflamación, desde la vagina al interior de la cavidad amniótica, probablemente por mejoría de los mecanismos inmunológicos del cérvix. Esta teoría está sustentada por el siguiente estudio. Mujeres embarazadas con insuficiencia cervical (incluye pacientes con cervix $<$ de $25 \mathrm{~mm}$ ) en comparación con gestantes normales, tuvieron concentraciones elevadas de citoquinas pro-inflamatorias IL-1b, IL-6, IL-8 IFN gama en el fluido cérvico-vaginal y no en la sangre, que disminuyeron significativamente con la colocación de cerclaje. Sugiere que las pacientes con insuficiencia cervical tienen alteración del sistema inmune innato vaginal y el cerclaje ayuda reduciendo la inflamación local cérvico-vaginal ${ }^{77}$.

Se recomienda la técnica de MacDonald con material de sutura monofilamento (p.ej. Ethibond ${ }^{\circledR}$ ) u otra y no con la cinta multifilamento (p.ej. Mersilene ${ }^{\circledR}$ ) u otra, la que suele infectarse y mantener la infección. La cinta multifilamento usada en mujeres embarazadas con riesgo de infección aumenta la posibilidad de resultado adverso por esta causa ${ }^{78}$. Debe hacerse en el primer trimestre y no esperar el acortamiento cervical ya que el aumento de citoquinas pro-inflamatorias existente en el flujo cérvicovaginal de estas pacientes, aumenta el riesgo de aborto de segundo trimestre y de $\mathrm{PP}^{26}$. Recomendación $\mathrm{B}^{70}$.

Es necesario hacer nuevos trabajos prospectivos, randomizados, con mayor casuística para confirmar el beneficio del cerclaje profiláctico en pacientes con antecedente de abortos de segundo trimestre y/o PPs por IBA, demostrados por biopsia placentaria y sin hijos vivos.

\section{Cerclaje terapéutico en pacientes con antecedente} de abortos de segundo trimestre y/o PPs por IBA, demostrados por biopsia placentaria, con hijos vivos y acortamiento cervical $<$ de $25 \mathrm{~mm}$

Este criterio se basa en la recomendación del metaanálisis ${ }^{74}$ que demostró que, en gestantes con embarazos únicos, PPs previos y longitud cervical $<25 \mathrm{~mm}$, el cerclaje previno el nacimiento prematuro, redujo la mortalidad perinatal y la morbilidad compuesta. Recomendación $\mathrm{A}^{70}$. Como evidencia para esta indicación, se aplica el criterio anterior que el cerclaje posiblemente reduciría la inflamación cervical y la $\mathrm{CH}$ en la población con riesgo de infección. Es necesario hacer trabajos prospectivos randomizados para confirmar esta aseveración en mujeres embarazadas con riesgo de IBA.

\section{Probióticos desde el primer trimestre del embarazo} en la paciente con riesgo de IBA y con flujo vaginal que demuestre ausencia o carencia de lactobacilos El beneficio de los probióticos se demuestra por las siguientes evidencias. El empleo de un probiótico oral conteniendo Lactobacilos rhamnosus GR1 junto con Lactobacilos reuteri $\mathrm{RC}-14$ versus placebo, restauró la microbiota vaginal normal $\mathrm{p}<0,001$, en pacientes ginecológicas con VB y con otras infecciones, usado durante seis semanas. Durante las siguientes seis semanas post tratamiento, el seguimiento demostró microbiota vaginal normal $^{79}$.

La RSC encontró que el uso de probiótico en la población general, oral o vaginal de L. rhamnosus GG, Bifidobacterium lactis BB12 o L. johnsonii redujo significativamente la infección genital, pero no disminuyó PP ni morbimortalidad perinatal ${ }^{80}$.

La evidencia no apoya actualmente el uso de probióticos durante el embarazo para reducir el PP. Es necesario hacer trabajos prospectivos, randomizados, con probióticos usados como única indicación en mujeres embarazadas con riesgo de IBA para investigar si reduce el PP. Sin embargo, se recomienda su uso para disminuir la frecuencia de infecciones vaginales y mantener la microbiota normal (Recomendación $\mathrm{A}^{70}$ ). Sugerimos el uso profiláctico de probióticos en mujeres embarazadas con riesgo de IBA, usuarias o no de cerclaje, con flujo vaginal que demuestre ausencia o disminución de lactobacilos, desde el término del primer trimestre, para impedir o atenuar la presentación de ICV. Las pacientes con riesgo de infección ascendente pueden carecer de lactobacilos desde el primer trimestre y habitualmente muestran inflamación y alteración del sistema inmune vaginal que incrementan $\mathrm{VB}$, VA y el $\mathrm{PP}^{26,34}$. El probiótico sería tratamiento coadyuvante en la prevención del PP. Recomendamos el probiótico de uso oral que contiene $L$. rhamnosus GR- $1^{\circledR}$, L. reuteri $\mathrm{RC}-14^{\circledR}$ o Bifidobacterium lactis $\mathrm{Bi}-07^{\circledR}$.

\section{Progesterona vaginal desde el término del primer trimestre en la paciente con riesgo de IBA, para mejorar la inmunidad vaginal}

La progesterona vaginal usada en mujeres embarazadas con cuello uterino $<$ de $25 \mathrm{~mm}$ en el segundo trimestre, medido por ultrasonografía, disminuye el riesgo de PP y mejora los resultados perinatales en gestaciones únicas sin ningún efecto perjudicial demostrable sobre el neurodesarrollo infantil ${ }^{81}$. Recomendación $\mathrm{A}^{70}$. Son variados los mecanismos postulados para explicar este beneficio. Uno de ellos es que la progesterona vaginal tiene efectos anti-inflamatorios locales en la interfase materno-fetal y en el cuello uterino, protegiendo contra el PP inducido en ratas por LPS de E. coli ${ }^{82}$.

Otras evidencias que sugieren que la progesterona vaginal mejora la inmunidad local son: a) La progesterona P4 inhibe in vitro la producción de IL-8 en células vaginales y cervicales producida por E. coli, G. vaginalis y $U$. urealyticum (bacterias asociadas con parto prematuro) ${ }^{83}$. 
b) Tiene un efecto inmunomodulador en la expresión de las citoquinas, FNT $\alpha$, IL-1b, IL-6, Il-8, IL-10 y MMP-9, al adicionar $\mathrm{P} 4$ a membranas corioamnióticas estimuladas con polisacáridos in vitro ${ }^{84}$.

Recomendamos la progesterona natural micronizada vaginal desde el término del primer trimestre y hasta las 37 semanas en pacientes en que no se usó cerclaje (Recomendación $\mathrm{A}^{70}$ ) y en aquéllas con cerclaje en que el tratamiento con probióticos no logra normalizar la microbiota vaginal ${ }^{26}$. Es necesario hacer trabajos prospectivos, randomizados, con progesterona usada como única indicación en mujeres embarazadas con riesgo de IBA, para investigar si reduce el PP por esta causa.

\section{Control metabólico de la diabetes mellitus (primer trimestre) o DG (segundo trimestre) para mejorar la inmunidad vaginal}

La recomendación del control metabólico de la diabetes pre o gestacional, se apoya en que las mujeres embarazadas con esta patología aumentan el riesgo de ICV y de bacteriuria, especialmente por SGB. La elevación de glicemia incrementa la frecuencia e intensidad de estas infecciones y dificulta el tratamiento por el aumento de la resistencia microbiana ${ }^{64}$.

\section{Control de peso en pacientes obesas para mejorar la inmunidad vaginal desde el primer trimestre}

Mientras mayor es la obesidad (IMC $>$ de $40 \mathrm{~kg} / \mathrm{m}^{2}$ ), aumentan los resultados adversos, incluido PP por IBA, por deterioro de la respuesta inmune innata vaginal $y$ adaptativa e inflamación sistémica. El mantener o subir moderadamente el peso durante el embarazo significa menor exposición al riesgo de infección por mejoría o menor deterioro del sistema inmune ${ }^{66-68}$.

Las diferentes recomendaciones para la prevención del PP por IBA en embarazo único, el nivel de atención de salud, la edad del embarazo y en que las pacientes deben ejecutarse estas acciones preventivas, se resumen en la Tabla 1.

Se concluye que a pesar de que se requieren más evidencias con estudios multicéntricos, randomizados, para demostrar la validez de nuestras recomendaciones, con las pruebas presentadas, basadas en fisiopatología e inmunología de la infección vaginal ascendente en la mujer embarazada con riesgo de infección, se puede pensar que el PP por IBA se conseguirá reducir. La comprensión actual de la IBA como principal factor de riesgo o condición obstétrica responsable del PP, la aplicación en este grupo de una serie de medidas eficientes

Tabla 1. Recomendaciones para la prevención del parto prematuro por infección bacteriana ascendente (IBA) en embarazo único

1. Detección de la población embarazada con riesgo de IGU durante el primer trimestre en nivel primario de atención de salud, mediante factores de riesgo de IBA

MANEJO de la paciente con riesgo de IGU que se detalla:

2. Pesquisa y tratamiento de ICV e ITU en todas las pacientes. En nivel primario pesquisa y en nivel secundario pesquisa y tratamiento. Toma de flujo vaginal + SGB y urocultivo durante el primer trimestre, entre las 22 y 24 semanas y entre las 28 y 30 semanas

3. Cerclaje profiláctico en pacientes con antecedente de abortos de segundo trimestre y/o partos prematuros por IBA, demostrados por biopsia placentaria y sin hijos vivos, realizado en el primer trimestre sin acortamiento cervical

4. Cerclaje terapéutico en pacientes con antecedente de abortos de segundo trimestre y/o partos prematuros por IBA, demostrados por biopsia placentaria, con hijos vivos y acortamiento cervical < de $25 \mathrm{~mm}$

Las pacientes con cerclaje y aquéllas sin abortos de segundo trimestre y/o partos prematuros por IBA, para mejorar la inmunidad vaginal deben seguir en el nivel secundario las recomendaciones del punto 5 al 8

5. Probióticos desde el primer trimestre del embarazo en todas las pacientes con microbiota vaginal que demuestre ausencia o carencia de lactobacilos

6. Progesterona vaginal desde el primer trimestre y hasta las 37 semanas en pacientes con microbiota anormal persistente

7. Control metabólico de la diabetes mellitus (primer trimestre) o diabetes gestacional ( ${ }^{\circ}$ trimestre)

8. Control de peso en pacientes obesas desde el primer trimestre

IGU: infección genito-urinaria. ICV: infección cérvico-vaginal. ITU: infección del tracto urinario. SGB: estreptococo grupo B (Streptococcus agalactiae). 
demostradas por la evidencia, como pesquisa rutinaria y tratamiento de las infecciones del tracto génito-urinario, cerclaje profiláctico o terapéutico, uso de probióticos, de progesterona, control metabólico de la diabetes mellitus y gestacional y del peso de la obesa, intervenciones que mejoran la inmunidad innata vaginal, permiten predecir reducción en los hospitales públicos de Chile del PP por IBA y de sus consecuencias, morbimortalidad perinatal, secuelas a largo plazo y costos elevados asociados.

\section{Resumen}

El parto prematuro (PP) es el principal contribuyente de la morbilidad/mortalidad perinatal. A pesar del conocimiento de los factores de riesgo y de la introducción de intervenciones médicas destinadas a la prevención del nacimiento prematuro, su frecuencia ha aumentado.
La infección bacteriana ascendente (IBA) es la condición obstétrica más frecuente asociada al PP ocasionando un importante resultado perinatal adverso en un hospital público de Chile. Esta revisión muestra la asociación entre PP e IBA, analiza la fisiopatología y la inmunología de las infecciones vaginales en la mujer embarazada susceptible, como asimismo la aplicación en este grupo de medidas con evidencia clínica que han demostrado ser eficientes, tales como la pesquisa rutinaria y el tratamiento de las infecciones genitourinarias (IGU), el cerclaje profiláctico o terapéutico, uso de probióticos, de progesterona vaginal, control metabólico de la diabetes mellitus y del peso de la obesa. El tratamiento de las IGU, conjuntamente con el uso de intervenciones que mejoran la inmunidad vaginal en la población de riesgo, permiten predecir una reducción del PP por IBA, de sus consecuencias inmediatas y de largo plazo y costos asociados elevados, con el consiguiente beneficio de la salud pública de Chile.

\section{Referencias bibliográficas}

1.- Beck S, Wojdyla D, Say L, Beltran AL, Merialdi M, Requejo J H, et al. The worldwide incidence of preterm birth: a systematic review of maternal mortality and morbidity. Bull World Health Org 2010; 88:31-38. doi: 10.2471/ BLT.08.062554.

2.- Goldenberg R L, Culhane J F, Iams J D, Romero R. Epidemiology and causes of preterm birth. Lancet 2008; 371:75-84. doi: 10.1016/S0140-6736(08)60074-4.

3.- Iams J D. Prevention of preterm parturition. $\mathrm{N}$ Engl J Med 2014; 370: 254-61. doi: 10.1056/ NEJMcp1103640.

4.- Chang H H, Larson J, Blencowe H, Spong C Y, Howson C P, Cairns-Smith S, et al. Preventing preterm birth: analysis of trends and potential reductions with interventions in 39 countries with very high human development index. Lancet 2013; 381: 223-34. doi: 10.1016/S01406736(12)61856-X.

5.- MacDorman M F, Mathews T J. BirthStats: percentage of preterm births, United States and selected European countries, 2004. Birth 2010; 37: 168. doi: 10.1111/j.1523536X.2010.00397.x

6.- Ananth C V, Vintzileos A M. Epidemiology of preterm birth and its clinical subtypes. $\mathrm{J}$ Matern Fetal Neonatal Med 2006; 19: 773-82. doi: 10.1080/14767050600965882.

7.- Ovalle A, Kakarieka E, Rencoret G, Fuentes A, del Río M J, Morong C, et al. Risk factors for preterm deliveries in a public hospital. Rev Med Chil 2012; 140: 19-29. doi: /S003498872012000100003

8.- Ministerio de Salud. Gobierno de Chile. Guía Clínica Prevención del Parto prematuro.
Santiago. MINSAL, 2010. ISBN: 978-9568823-84-9.

9.- Ministerio de Salud. Gobierno de Chile. Guía Perinatal 2015, Capítulo XXI Parto Prematuro p. 276-290. Santiago MINSAL 2015. ISBN: 978-956-348-076-4.

10.- Sentilhes L, Sénat M V, Ancel P Y, Azria E, Benoist G, Blanc J, et al. Prevention of spontaneous preterm birth: Guidelines for clinical practice from the French College of Gynaecologists and Obstetricians (CNGOF). Eur J Obstet Gynecol Reprod Biol 2017; 210 : 217-24. doi: 10.1016/j.ejogrb.2016.12.035.

11.- Keelan J A, Newnham J P. Recent advances in the prevention of preterm birth. F1000Res. 2017; 6: F1000 Faculty Rev-1139. doi:10.12688/f1000research.11385.1.

12.- Ovalle A, Kakarieka E, Díaz M, García Huidobro T, Acuña J, Fuentes A, et al Mortalidad perinatal en el parto prematuro entre 22 y 34 semanas en un hospital público de Santiago, Chile Rev Chil Obstet Ginecol 2012; 77: 263-70. http://dx.doi.org/10.4067/S071775262012000400005.

13.- Ovalle A, Fuentes A, Chacón V, Espinoza C, González R, Kakarieka E, et al. A new fetal death classification system. Rev Med Chile 2016; 144: 1020-8. doi: 10.4067/S003498872016000800009 .

14.- Ovalle A, Gómez R, Martínez M A, Kakarieka E, Fuentes A, Aspillaga C, et al. Invasión microbiana de la cavidad amniótica en la rotura de membranas de pretérmino. Resultados materno-neonatales y patología placentaria según microorganismo aislado. Rev Med Chil 2005; 133: 51-61. http://dx.doi.org/10.4067/ S0034-98872005000100007.

15.- Ovalle A, Martínez M A, Kakarieka M
E, Gómez R, Torres J, Fuentes A, et al. Histopatología de la placenta en la rotura prematura de membranas de pretérmino. Relación con la microbiología aislada y con los resultados materno-neonatales. Rev Med Chile 1998; 126: 930-42. PMID: 9830745.

16.- Gómez R, Romero R, Nien JK, Medina L, Carstens M, Kim Y M, et al. Idiopathic vaginal bleeding during pregnancy as the only clinical manifestation of intrauterine infection. J Matern Fetal Neonatal Med. 2005;18: 31-7. doi: 10.1080/14767050500217863.

17.- Romero R, González R, Sepúlveda W, Brandt F, Ramírez M, Sorokin Y, et al. Infection and labor. VIII. Microbial invasion of the amniotic cavity in patients with suspected cervical incompetence: prevalence and clinical significance. Am J Obstet Gynecol 1992; 167: 1086-91. PMID: 1415396.

18.- Vaisbuch E, Hassan S S, Mazaki-Tovi S, Nhan-Chang C L, Kusanovic J P, Romero R, et al. Patients with an asymptomatic short cervix $(<15 \mathrm{~mm})$ have a high rate of subclinical intraamniotic inflammation: implications for patient counseling. Am J Obstet Gynecol 2010; 202: 433.e1-8. doi: 10.1016/j.ajog.2010.02.007.

19.- Ravel J, Gajer P, Abdo Z, Schneider, G M, Koenig, S S, McCulle S L, et al. Vaginal microbiome of reproductive-age women. Proc Natl Acad Sci 2011; 108: 4680-87. doi: 10.1073/pnas.1002611107.

20.- Donati L, Di Vico A, Nucci M, Quagliozzi L, Spagnuolo T, Labianca A, et al. Vaginal microbial flora and outcome of pregnancy. Arch Gynecol Obstet 2010; 281: 589-600. doi: 10.1007/s00404-009-1318-3.

21.- Aroutcheva A, Gariti D, Simon M, Shott S, Faro J, Simoes J A, et al. Defense factors of 
vaginal lactobacilli. Am J Obstet Gynecol 2001; 185: 375-9. doi: 10.1067/mob.2001.115867.

22.- Haya J, García A, López-Manzanara C, Balawi M, Haya L. Importance of lactic acid in maintaining vaginal health: A review of vaginitis and vaginosis etiopathogenic bases and a proposal for a new treatment. Open J Obstet Gynecol 2014; 4: 787-99. doi.org/10.4236/ojog.2014.413109

23.- Hay P E, Lamont R F, Taylor-Robinson D, Morgan D J, Ison C, Pearson J. Abnormal bacterial colonisation of the genital tract and subsequent preterm delivery and late miscarriage. Br Med J 1994; 308: 295-8. PMCID: PMC2539287.

24.- Hillier S L, Nugent R P, Eschenbach D A, Krohn M A, Gibbs R S, Martin D H, et al. Association between bacterial vaginosis and preterm delivery of a low-birth-weight infant. $\mathrm{N}$ Engl J Med 1995; 333: 1737-42. doi=10.1.1.46 4.5929\&rep=rep1\&type $=$ pdf.

25.- Ovalle A, Martínez M A, Giglio M S, Fuentes A, Villablanca E. Microbiología aislada en la rotura prematura de membranas de pretérmino. Relación con morbilidad infecciosa materna neonatal e intervalo rotura de membranas-parto. Rev Chil Obstet Ginecol 1995; 60: 252-62.

26.- Donders G G, Van Calsteren K, Reybrouck B G, Van den Bosch R R, Van Lierde R I. Predictive value for preterm birth of abnormal vaginal flora, bacterial vaginosis and aerobic vaginitis during the first trimester of pregnancy. Br J Obstet Gynecol 2009; 116: 1315-24. doi: 10.1111/j.1471-0528.2009.02237.x

27.- Ovalle A, Martínez M A. Capítulo 30: Infección genital. Guzmán E, Editor. Selección de temas en Gineco-obstetricia, 1a ed. Editorial Publimpacto; 2007, p. 875-923.

28.- Goldenberg R L, Klebanoff M A, Nugent R, Krohn M A, Hillier S, Andrews W W. Bacterial colonization of the vagina during pregnancy in four ethnic groups. Vaginal Infections and Prematurity Study Group. Am J Obstet Gynecol 1996; 174: 1618-21. PMID:9065140.

29.- Hickey R J, Zhou X, Pierson J D, Ravel J, Forney L J. Understanding vaginal microbiome complexity from an ecological perspective. Transl Res 2012; 160: 267-82. doi: 10.1016/j. trs1.2012.02.008.

30.- Ministerio de Salud. Gobierno de Chile. Guía Perinatal 2015, Capítulo XVII Infecciones cervicovaginales. Infecciones urinarias durante el embarazo. P. 206-23. Santiago Minsal 2015. ISBN: 978-956-348-076-4.

31.- Castro J, Alves P, Sousa C, Cereija T, Franca C, Jefferson $\mathrm{K} \mathrm{K}$, et al. Using an in-vitro biofilm model to assess the virulence potential of bacterial vaginosis or non-bacterial vaginosis Gardnerella vaginalis isolates. Sci Rep 2015; 5 : 11640. doi: 10.1038/srep11640.

32.- Schwebke J R, Muzny C A, Josey W E. Role of Gardnerella vaginalis in the pathogenesis of bacterial vaginosis: a conceptual model. J Infect Dis 2014; 210: 338-43. doi: 10.1093/infdis/ jiu089.

33.- Srinivasan S, Hoffman N G, Morgan M T, Matsen F A, Fiedler T L, Hall R W, et al. Bacterial communities in women with bacterial vaginosis: high resolution phylogenetic analyses reveal relationships of microbiota to clinical criteria. PLoS ONE 2012; 7(6):e37818. doi:10.137/journal.pone 0037818 .

34.- Ovalle A, Martínez M A, Villagra E, Roncone E Sandoval R, Silva R. Flora microbiana genital en embarazadas con y sin riesgo de infección. Rev Chil Obstet Ginecol 1996; 61: 5-11

35.- Macones G A, Parry S, Elkousy M, Clothier B, Ural S H, Strauss J F 3rd. A polymorphism in the promoter region of TNF and bacterial vaginosis: preliminary evidence of geneenvironment interaction in the etiology of spontaneous preterm birth. Am J Obstet Gynecol 2004; 190: 1504-8. DOI: 10.1016/j. ajog.2004.01.001.

36.- Donders G G, Vereecken A, Bosmans E, Dekeersmaecker A, Salembier G, Spitz B. Definition of a type of abnormal vaginal flora that is distinct from bacterial vaginosis: aerobic vaginitis. Br J Obstet Gynecol 2002; 109: 34 43. PMID: 11845812.

37.- Seale A C, Bianchi-Jassir F, Russell N J, Kohli-Lynch M, Tann C J, et al Estimates of the burden of group B streptococcal disease worldwide for pregnant women, Stillbirths, and Children. Clin Infect Dis 2017; 65 (Suppl 2): S200-19. doi: 10.1093/cid/cix664.

38.- Ovalle A, García M, Oda F, Alvarado S, Martínez M A. Meningitis neonatal precoz causada por transmisión vertical de Escherichia coli productora de beta-lactamasa de espectro extendido en parto prematuro con rotura prematura de membranas. Rev Chil Obstet Ginecol 2017; 82: 621-5. http://dx.doi. org/10.4067/S0717-75262017000600621.

39.- Amabebe E, Chapman D R, Stern V L, Stafford G, Anumba D O C. Mid-gestational changes in cervicovaginal fluid cytokine levels in asymptomatic pregnant women are predictive markers of inflammation-associated spontaneous preterm birth. J Reprod Immunol 2018; 126: 1-10. doi: 10.1016/j.jri.2018.01.001

40.- Ovalle A, Romero R, Gómez R, Martínez M A, Nien J K, Figueroa J, et al. Antibiotics administration to patients with preterm labor an intact membranes: is there a beneficial effects in patients with endocervical inflammation? J Matern Fetal Neonatal Med 2006; 19: 453-64. doi: 10.1080/14767050600852668.

41.- Nugent R P, Krohn M A, Hillier S L. Reliability of diagnosing bacterial vaginosis is improved by standardized method of gram stain interpretation. J Clin Microbiol 1991; 29: 297301. PMCID: PMC269757.

42.- Ison C A, Hay P E. Validation of a simplified grading of Gram stained vaginal smears for use in genitourinary medicine clinics. Sex Transm Infect 2002;78: 413-5. PMCID: PMC1758337.

43.- Farr A, Kiss H, Hagmann M, Machal S, Holzer I, Kueronya V, et al. Role of Lactobacillus species in the intermediate vaginal flora in early pregnancy: A retrospective cohort study. PLoS ONE 2015; 10 (12): e0144181. doi:10.1371/journal.pone.0144181.

44.- Taylor-Robinson D, Lamont RF. Mycoplasmas in pregnancy. Br J Obstet Gynecol 2011; 118: 164-74. doi: $10.1111 / \mathbf{j} .1471$ 0528.2010.02766.x

45.- Luton D, Ville Y, Luton-Sigy A, Cousin C, Narraido B, Fassasi-Jarretou A, et al. Prevalence and influence of Mycoplasma hominis and Ureaplasma urealyticum in 218 African pregnant women and their infants. Eur J Obstet Gynecol Reprod Biol 1994; 56: 95101. PMID: 7805974.

46.- Ovalle A, Martínez M A, Gómez R, Sáez J, Menares I, Aspillaga C, et al. Parto prematuro con membranas intactas. Microbiología del líquido amniótico y tracto genital inferior y su relación con los resultados materno neonatales. Rev Med Chile 2000; 128: 985-95. http://dx.doi.org/10.4067/S003498872000000900005

47.- Schelonka R L, Katz B, Waites K B, Benjamin D K Jr. Critical appraisal of the role of Ureaplasma in the development of bronchopulmonary dysplasia with metaanalytic techniques. Pediatr Infect Dis J 2005; 24: 1033-9. PMID: 16371861.

48.- Raynes Greenow C H, Roberts C L, Bell J C, Peat B, Gilbert G L, et al. Antibiotics for ureaplasma in the vagina in pregnancy. Cochrane Database Syst Rev 2011; (9): CD003767. doi: 10.1002/14651858.CD003767. pub3. Review.

49.- Rodríguez N, Fernández C, Zamora Y, Berdasquera D, Rivera J A. Detection of $U$. urealyticum and $U$. parvum in amniotic fluid: association with pregnancy outcomes. J Matern Fetal Neonatal Med 2011; 24: 47-50. doi: 10.3109/14767058.2010.482609.

50.- Ovalle A, Gómez R, Martínez M A, Rubio R, Fuentes, Romero R, et al. Antibiotic therapy in patients with preterm premature rupture of membranes: a prospective, randomized, placebo controlled study with microbiological assessment of the amniotic cavity and lower genital tract. Prenatal Neonatal Med 1997; 2: 213-22.

51.- Lamont R F, Nhan-Chang C L, Sobel J D, Workowski K, Conde-Agudelo A, Romero R. Treatment of abnormal vaginal flora in early pregnancy with clindamycin for the prevention of spontaneous preterm birth: a systematic review and meta-analysis. Am J Obstet Gynecol 2011; 205: 177-90. doi: 10.1016/j. ajog.2011.03.047. 
52.- Ovalle A. Espectro clínico de las infecciones por Chlamydia trachomatis en mujeres. Rev Chilena Infectol 1997; 15: 9-17.

53.- Ovalle A, Silva O, Herrera R, González E, Marín J. Antecedentes mórbidos de riesgo y predicción de la infección génitourinaria durante el embarazo. Rev Chil Obstet Ginecol 1989; 54: 341-7. PMID: 2485368.

54.- Horne A W, Stock S J, King A E. Innate immunity and disorders of the female reproductive tract. Reproduction 2008; 135 : 739-49. doi: 10.1530/REP-07-0564.

55.- Frew L, Stock S J. Antimicrobial peptides and pregnancy. Reproduction 2011; 141: 725-35. doi: 10.1530/REP-10-0537.

56.- Marconi C, Donders G G, Parada C M, Giraldo P C, da Silva M G. Do Atopobium vaginae, Megasphaera sp. and Leptotrichia $\mathrm{sp}$ change the local innate immune response and sialidase activity in bacterial vaginosis? Sex Transm Infect 2013; 89: 167-73. doi: 10.1136/ sextrans-2012-050616.

57.- Valore E V, Wiley D J, Ganz T. Reversible deficiency of antimicrobial polypeptides in bacterial vaginosis. Infect Immun 2006; 74 : 5693-702. doi: 10.1128/IAI.00524-06.

58.- Donders G, Bellen G, Rezeberga D. Aerobic vaginitis in pregnancy. Br J Obstet Gynecol 2011; 118: 1163-70. doi: 10.1111/j.14710528.2011.03020.x

59.- Mitchell C, Gottsch M L, Liu C, Fredricks D $\mathrm{N}$, Nelson D B. Associations between vaginal bacteria and levels of vaginal defensins in pregnant women. Am J Obstet Gynecol 2013; 208:132.e1-7. doi: 10.1016/j.ajog.2012.11.019.

60.- Xu J, Holzman C B, Arvidson C G, Chung H, Goepfert A R. Midpregnancy vaginal fluid defensins, bacterial vaginosis, and risk of preterm delivery. Obstet Gynecol 2008; 112: 524-31. doi: 10.1097/AOG.0b013e318184209b.

61.- Soto E, Espinoza J, Nien J K, Kusanovic J P, Erez O, Richani K, Santolaya-Forgas J, Romero, et al. Human beta-defensin-2 a natural antimicrobial peptide present in amniotic fluid participates in the host response to microbial invasion of the amniotic cavity. $\mathrm{J}$ Matern Fetal Neonatal Med 2007; 20: 15-22. doi: 10.1080/14767050601036212.

62.- Heine R P, Wiesenfeld H, Mortimer L, Greig P C. Amniotic fluid defensins: potential markers of subclinical intrauterine infection. Clin Infect Dis 1998; 27: 513-8. PMID: 9770150.

63.- King A E, Kelly R W, Sallenave J M, Bocking A D, Challis J R. Innate immune defenses in the human uterus during pregnancy. Placenta 2007; 28: 1099-106. DOI: 10.1016/j. placenta.2007.06.002.

64.- Alvarez J R, Fechner A J, Williams S F, Ganesh V L, Apuzzio J J. Asymptomatic bacteriuria in pregestational diabetic pregnancies and the role of group B streptococcus. Am J Perinatol 2010; 27: 231-4. doi: 10.1055/s-0029-1239485.

65.- Christian L M, Franco A, Iams J D, Sheridan J, Glaser R. Depressive symptoms predict exaggerated inflammatory responses to an in vivo immune challenge among pregnant women. Brain Behav Immun 2010; 24:49-53. doi: 10.1016/j.bbi.2009.05.055.

66.- Ovalle A, Martínez M A, Fuentes A, Marques $\mathrm{X}$, Vargas F, Vergara P, et al. Obesity, a risk factor for ascending bacterial infection during pregnancy. Rev Med Chile 2016; 144:476-82. https://scielo.conicyt.cl/pdf/rmc/v144n4/art08. pdf.

67.- Sen S, Iyer C, Klebenov D, Histed A, Aviles J A, Meydani S N. Obesity impairs cell- mediated immunity during the second trimester of pregnancy. Am J Obstet Gynecol 2013; 208 (2):139. e1-8. doi: 10.1016/j. ajog.2012.11.004.

68.- Shapiro H, Lutaty A, Ariel A. Macrophages, meta-inflammation, and immuno-metabolism. ScientificWorldJournal 2011; 11: 2509-29. doi: 10.1100/2011/397971.

69.- Bender Ignacio R A, Madison A T, Moshiri A, Weiss N S, Mueller B A. A Population-based study of perinatal infection risk in women with and without systemic lupus erythematosus and their infants. Paediatr Perinat Epidemiol 2018, 32:81-89. doi: 10.1111/ppe.12430.

70.- Manterola C, Asenjo-Lobos C, Otzen T. Hierarchy of evidence: levels of evidence and grades of recommendation from current use. Rev Chilena Infectol 2014; 31:705-18. doi: 10.4067/S0716-10182014000600011.

71.- Sangkomkamhang U S, Lumbiganon P, Prasertcharoensuk W, Laopaiboon M. Antenatal lower genital tract infection screening and treatment programs for preventingpreterm delivery.Cochrane Database Syst Rev 2015;(2): CD006178.

72.- Smaill F M, Vazquez J C. Antibiotics for asymptomatic bacteriuria in pregnancy. Cochrane Database Syst Rev 2015; (8): CD000490.

73.- Ovalle A, Sáez J, Martínez M A, Cona E, Bueno F, Leyton H, et al. Infección cérvicovaginal en la pielonefritis aguda del embarazo. Relación con los resultados del tratamiento antibiótico y con la contractilidad uterina anormal. Rev Chil Obstet Ginecol 2000; 65: 346-54

74.- Berghella V, Rafael T J, Szychowski J M, Rust O A, Owen J. Cerclage for short cervix on ultrasonography in women with singleton gestations and previous preterm birth: a metaanalysis. Obstet Gynecol 2011; 117: 663-71. PMID: 21446209.

75.- Alfirevic Z, Stampalija T, Medley N. Cervical stitch (cerclage) for preventing preterm birth in singleton pregnancy. Cochrane Database Syst Rev 2017; 6: CD008991

76.- Ovalle A, Valderrama O, Rencoret G, Fuentes A, Kakarieka E, Martínez M A, et al. Cerclaje profiláctico en mujeres con nacimientos prematuros espontáneos previos asociados con infección bacteriana ascendente. Rev Chil Obstet Ginecol 2012; 77: 98-105. https://scielo. conicyt.cl/pdf/rchog/v77n2/art04.pdf.

77.- Monsanto SP, Daher S, Ono E, Pendeloski KPT, Trainá É, Mattar R, et al. Cervical cerclage placement decreases local levels of proinflammatory cytokines in patients with cervical insufficiency. Am J Obstet Gynecol 2017; 217: 455. e1-455.e8. doi: 10.1016/j. ajog.2017.06.024.

78.- Israfil-Bayli F, Toozs-Hobson P, Lees C, Slack M, Ismail K M. Pregnancy outcome after elective cervical cerclage in relation to type of suture material used. Med Hypotheses 2013; 81: 119-21. doi: 10.1016/j.mehy.2013.04.00.

79.- Vujic G, Jajac Knez A, Despot Stefanovic V, Kuzmic Vrbanovic V. Efficacy of orally applied probiotic capsules for bacterial vaginosis and other vaginal infections: a double-blind, randomized, placebo-controlled study. Eur J Obstet Gynecol Reprod Biol 2013; 168:75-9. doi: 10.1016/j.ejogrb.2012.12.031

80.- Othman M, Neilson J P, Alfirevic Z. Probiotics for preventing preterm labour. Cochrane Database Syst Rev 2007; (1): CD005941.

81.- Romero R, Conde-Agudelo A, Da Fonseca E, O’Brien JM, Cetingoz E, Nicolaides KH, et al. Vaginal progesterone for preventing preterm birth and adverse perinatal outcomes in singleton gestations with a short cervix: a metaanalysis of individual patient data. Am J Obstet Gynecol 2018; 218: 161-80. doi: 10.1016/j. ajog.2017.11.576.

82.- Furcron A E, Romero R, Plazyo O, Unkel R, $\mathrm{Xu}$ Y, Hassan S S, et al. Vaginal progesterone, but not $17 \alpha$-hydroxyprogesterone caproate, has antiinflammatory effects at the murine maternal-fetal interface. Am J Obstet Gynecol 2015; 213:846.e1-846.e19. doi: 10.1016/j. ajog.2015.08.010.

83.- Peltier M R, Berlin Y, Tee S C, Smulian J C. Does progesterone inhibit bacteria-stimulated interleukin-8 production by lower genital tract epithelial cells? J Perinat Med 2009; 37: 32833. doi: 10.1515/JPM.2009.064.

84.- Pineda-Torres M, Flores-Espinosa P, EspejelNunez A, Estrada-Gutiérrez G, Flores-Pliego A, Maida-Claros R, et al. Evidence of an immunosuppressive effect of progesterone upon in vitro secretion of proinflammatory and prodegradative factors in a model of choriodecidual infection. Br J Obstet Gynecol 2015; 122:1798-807. doi: 10.1111/14710528.13113 . 